\title{
Secrecy Analysis of Full-Duplex Power-Splitting SWIPT Networks with Artificial Noise
}

\author{
Xuanxuan Tang ${ }^{1}$, Wendong Yang ${ }^{1,2}{ }^{+}$, Yueming Cai ${ }^{1}$, Weiwei Yang ${ }^{1}$, Kuo Cao ${ }^{1}$ and Pan Yuan ${ }^{1}$ \\ ${ }^{1}$ College of Communications Engineering, PLA University of Science and Technology, Nanjing, China. \\ ${ }^{2}$ Key Lab of Broadband Wireless Communication and Sensor Network Technology (Nanjing University of \\ Post and Telecommunications), Ministry of Education, China
}

\begin{abstract}
This work conducts the secrecy analysis of full-duplex power-splitting simultaneous wireless information and power transfer networks with artificial noise. The exact closed-form expressions of the COP, SOP and RSP are derived, respectively, which are illustrated by numerical analysis. The results depict the significance of the power-splitting ratio and the transmit signal-to-noise ratio (SNR). To be specific, the secrecy performance is greatly improved with the increasing of the power-splitting ratio. In addition, it is observed that the security of the system will get enhanced with the increasing of the power-splitting ratio at first and then get reversed for a certain transmit SNR. Moreover, the value of the optimum transmit SNR changes with different transmit SNR.
\end{abstract}

Keywords: secure, SWIPT, full-duplex, artificial noise, power-splitting

\section{Introduction}

Due to the broadcast nature of wireless transmission, it is inherently easy to be intercepted by unauthorized users for wireless communication. As an emerging and promising technology, the physical layer security (PLS) tries to further enhance the security of wireless networks by exploiting physical channels, which is totally different from traditional cryptography technologies at higher layers. Since pioneered by Wyner [1] in 1975, numerous researches on PLS have been conducted under various wireless networks, such as cognitive radio (CR) networks [2], device-to-device (D2D) networks [3], and wireless sensor networks (WSN) [4].

Being an effective approach of signal processing to strengthen the security of wireless networks, the artificial noise (AN) has captured great interest of researchers [5]. The authors in [6] proposed a transmission protocol where the AN and the information-bearing signal are transmitted together by the transmitter to confuse the eavesdropper. In [7], a joint relay and jammer selection scheme was presented for improving the system secrecy under the effect of imperfect channel state information (CSI), where the AN was sent by a separate jammer.

On the other hand, energy harvesting with simultaneous wireless information and power transfer (SWIPT) has recently arisen as an appealing technique to tackle the energy efficiency of wireless communications, especially in some energy constrained scenarios [8]. In [9, 10], two time-switching (TS) SWIPT schemes were respectively studied with full-duplex (FD) relay harvesting the energy from the source which is then used to forward the data to the destination. Under a similar scenario, work [11] presented a FD SWIPT relaying scheme from a power-splitting (PS) perspective. Nonetheless, all the study in work [9-11] did not concern the security issue. Literature [12] addressed the security of a FD SWIPT system, where a wireless-powered full-duplex (FD) jammer is set to accumulate energy and then transmit AN to help improve

${ }^{+}$Corresponding author. Tel.: +8602580828018 ; fax: +8602580828018 .

E-mail address: ywd1110@163.com. 
the security. However, no published work has investigated the security of FD PS SWIPT Networks with Artificial Noise.

This work studies the secure transmission of FD PS SWIPT Networks with Artificial Noise. The exact closed-form expressions of the connection outage probability (COP), the secrecy outage probability (SOP) and the reliable-secure probability (RSP) are obtained, which depict the secrecy performance very well. The rest of this work are organised as follows: section 2 and 3 give the description of the system model and the transmission scheme respectively, section 4 conducts the secrecy analysis of the proposed system, section 5 validates the analysis with numerical simulations. Finally, section 6 summarises the whole paper.

\section{System Model}

We consider a point-to-point communication system, which consists of a source $S$, a destination $D$ and a potential eavesdropper $E$, as shown in Fig. 1. Specifically, $D$ is an energy-constrained node and is equipped with two antennas, denoted as $D_{1}$ and $D_{2}$, respectively. Thus, it can harvest energy and information with the $D_{1}$ from the wireless signals, and transmits AN to confuse $E$ via $D_{2}$ at the same time. Meanwhile, both $S$ and $E$ are equipped with a single antenna. Moreover, we assume that all the channels undergo quasi-static flat Rayleigh fading and Additive White Gaussian Noise (AWGN) with zero mean and variance $N_{0}$. That is to say, the channel coefficients stay constant within each transmission block of duration $T$, but change independently from one block to another ${ }^{1}$. Without loss of generality, let $h_{a b}$ represents the channel coefficients of link $a \rightarrow b$, where $a, b \in\left\{S, D_{1}, D_{2}, E\right\}$. Hence, the channel gain $\left|h_{a b}\right|^{2}$ is an exponentially distributed random variable with the mean $\bar{\gamma}_{a b}=E\left[\left|h_{a b}\right|^{2}\right]$, where $E[\mathrm{~g}]$ denotes the expectation operation. Specifically, we let $h_{I}=h_{D_{2} D_{1}}$, which represents the self-interference channel with the mean of $\bar{\gamma}_{I}$. The channel after self-interference cancellation (SIC) is defined as $h_{I}^{\prime}=h_{D_{2} D_{1}}^{\prime}$ with the mean of $\bar{\gamma}_{I}^{\prime}$. In the following section, we denote $g_{(\mathrm{g})}=\left|h_{(\mathrm{g})}\right|^{2}$ for the convenience of notation.

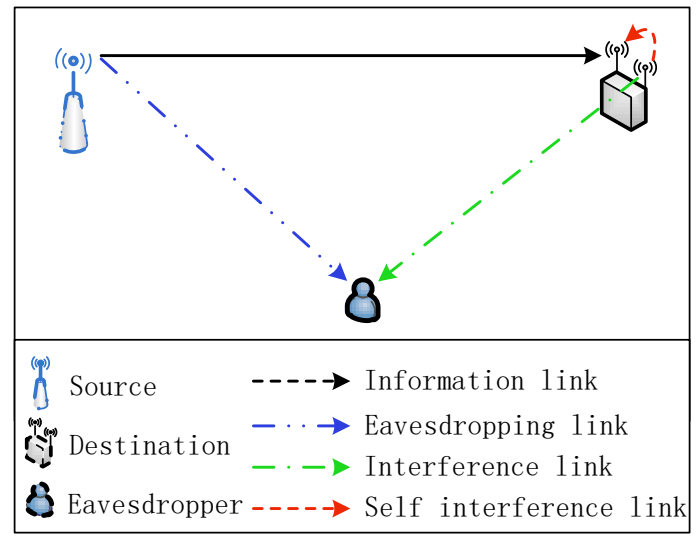

Fig. 1: System model.

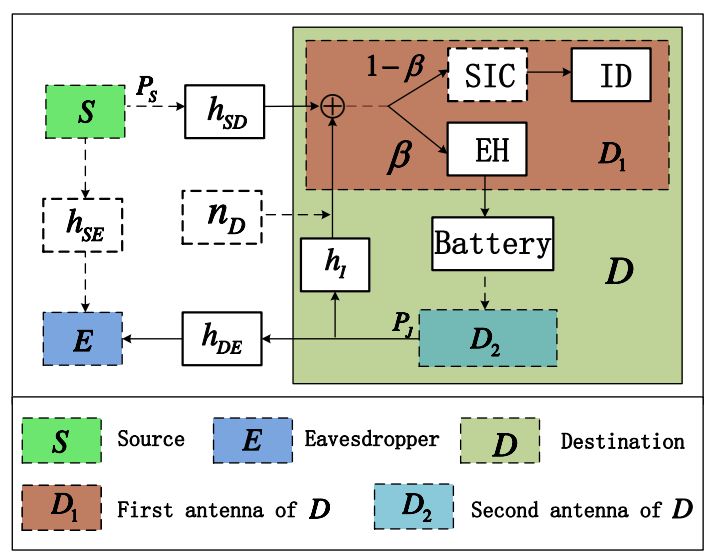

Fig. 2: Model of FD PS-SWIPT with AN.

\footnotetext{
${ }^{1}$ This common assumption has been widely adopted by numerous literatures on wireless communications, see $[2,9,11]$.
} 


\section{FD PS-SWIPT with AN Protocol}

The whole process of the transmission is illustrated as in Fig. 2. $S$ transmits its signal to $D$ which is received by its first antenna $D_{1}$. We note that, the signal from $S$ will also be received by $E$. Therefore, $D$ sends AN at the same time with its second antenna $D_{2}$ for safeguarding the secrecy information. As such, the signal received by $D_{1}$ and $E$ are as follows

$$
\begin{gathered}
y_{D}=\sqrt{P_{S}} h_{S D} x+\sqrt{P_{J}} h_{I} x_{J}+n_{D} \\
y_{E}=\sqrt{P_{S}} h_{S E} x+\sqrt{P_{J}} h_{D E} x_{J}+n_{E}
\end{gathered}
$$

where $P_{S}$ represents the transmit power of $S, x$ is the information-bearing signal with $E\left[|x|^{2}\right]=1 . P_{J}$ denotes the AN power of $D_{2}, x_{J}$ is the AN with $E\left[\left|x_{J}\right|^{2}\right]=1 . n_{D}$ and $n_{E}$ represent the AWGN at $D$ and $E$, respectively.

As depicted in system model, the PS-SWIPT protocol is adopted at $D$ for obtaining the information and collecting energy simultaneous. Therefore, the received energy at $D$ can be expressed by

$$
E_{D}=\eta \beta T\left(P_{S} g_{S D}+P_{J} g_{I}\right)
$$

where $\eta(0 \leq \eta \leq 1)$ is the energy conversion efficiency, $\beta(0 \leq \beta \leq 1)$ is the ratio of power split for energy harvesting (EH), and the part of power for information decoding (ID) is $(1-\beta)$. Hence, the signal-tointerference-plus-noise ratio (SINR) at $D$ and $E$ are given by

$$
\begin{gathered}
\gamma_{D}=\frac{(1-\beta) P_{S} g_{S D}}{P_{J} g_{9}+N_{0}}=\frac{(1-\beta) \gamma_{S} g_{S D}}{\gamma_{J} g_{9}+1} \\
\gamma_{E}=\frac{P_{S} g_{S E}}{P_{J} g_{D E}+N_{0}}=\frac{\gamma_{S} g_{S E}}{\gamma_{J} g_{D E}+1}
\end{gathered}
$$

where $\gamma_{S} @ \frac{P_{S}}{N_{0}}, \gamma_{J} @ \frac{P_{J}}{N_{0}}$ represent the transmit signal-to-noise ratio (SNR) of $S$ and $D$, respectively.

As $D$ is energy-constrained, $P_{J}$ is determined according to expression (6) so as to keep the energy balance

$$
P_{J}=\frac{E\left[E_{D}\right]}{T}
$$

which easily yields to

$$
P_{J}=\frac{\eta \beta \bar{\gamma}_{S D}}{1-\eta \beta \bar{\gamma}_{I}} P_{S}
$$

\section{Exact Secrecy Analysis}

In this section, the COP, SOP and RSP are investigated, whose definitions are given in [7].

\subsection{COP Analysis}

Theorem 1: The COP of the FD PS-SWIPT system with AN is given by

$$
P_{C O}=1-\frac{\bar{g}_{S D}(1-\beta)\left(1-\eta \beta \bar{\gamma}_{I}\right)}{\bar{g}_{I}^{\prime} \gamma_{t}^{t h} \eta \beta \bar{\gamma}_{S D}+(1-\beta)\left(1-\eta \beta \bar{\gamma}_{I}\right)} \exp \left(-\frac{\gamma_{t}^{\text {th }}}{\bar{g}_{S D}(1-\beta) \gamma_{S}}\right)
$$

where $\gamma_{t}^{\text {th }} @ 2^{R_{t}}-1$, and $R_{t}$ represents the transmission rate per channel use.

Proof: According to the definition in [7], the COP can be given by

$$
P_{C O}=\operatorname{Pr}\left[\gamma_{D}<\gamma_{t}^{t h}\right]=\operatorname{Pr}\left[\frac{(1-\beta) \gamma_{S} g_{S D}}{\gamma_{J} g_{I}^{\prime}+1}<\gamma_{t}^{t h}\right]
$$

Hence, equation (9) can be rewritten as

$$
P_{C O}=\int_{0}^{\infty} F_{S_{S D}}\left(\frac{\gamma_{t}^{\text {th }}\left(\gamma_{J} x+1\right)}{(1-\beta) \gamma_{S}}\right) f_{g^{\prime}}(x) d x
$$


Recalling that $g_{S D}$ and $g_{I}^{\prime}$ are both exponentially distributed random variables with the mean of $\bar{\gamma}_{S D}$ and $\bar{\gamma}_{I}^{\prime}$. By substituting the distributing properties we get

$$
P_{C O}=\int_{0}^{\infty}\left(1-\exp \left(-\frac{\gamma_{t}^{\text {th }}\left(\gamma_{J} x+1\right)}{\bar{g}_{S D}(1-\beta) \gamma_{S}}\right)\right) \frac{1}{\bar{g}_{I}^{\prime}} \exp \left(-\frac{x}{\bar{g}_{I}^{\prime}}\right) d x
$$

which yields to Theorem 1 with some simple calculations.

\subsection{SOP Analysis}

Theorem 2: The SOP of the FD PS-SWIPT system with AN is given by

$$
P_{S O}=\frac{\bar{g}_{S E}\left(1-\eta \beta \bar{\gamma}_{I}\right)}{\bar{g}_{D E} \gamma_{e}^{\text {th }} \eta \beta \bar{\gamma}_{S D}+\left(1-\eta \beta \bar{\gamma}_{I}\right)} \exp \left(-\frac{\gamma_{e}^{\text {th }}}{\bar{g}_{S E} \gamma_{S}}\right)
$$

where $\gamma_{e}^{\text {th }} @ 2^{R_{t}-R_{s}}-1$, and $R_{s}$ denotes the secrecy information rate per channel use.

Proof: Resorting to [7], the SOP is defined as

$$
P_{S O}=\operatorname{Pr}\left[\gamma_{E} \geq \gamma_{e}^{\text {th }}\right]=\operatorname{Pr}\left\lfloor\frac{\gamma_{S} g_{S E}}{\gamma_{J} g_{D E}+1} \geq \gamma_{e}^{\text {th }}\right\rfloor
$$

It is noted that (13) has the similar form with (9). Thereby, the expression in Theorem 2 can be easily obtained with the similar method.

\subsection{RSP Analysis}

Theorem 3: The RSP of the FD PS-SWIPT system with AN is given by

$$
P_{R S}=\frac{\bar{g}_{S D}(1-\beta)\left(1-\eta \beta \bar{\gamma}_{I}\right)}{\bar{g}_{I}^{\prime} \gamma_{t}^{\text {th }} \eta \beta \bar{\gamma}_{S D}+(1-\beta)\left(1-\eta \beta \bar{\gamma}_{I}\right)} \exp \left(-\frac{\gamma_{t}^{\text {th }}}{\bar{g}_{S D}(1-\beta) \gamma_{S}}\right)\left[1-\frac{\bar{g}_{S E}\left(1-\eta \beta \bar{\gamma}_{I}\right)}{\bar{g}_{D E} \gamma_{e}^{\text {th }} \eta \beta \bar{\gamma}_{S D}+\left(1-\eta \beta \bar{\gamma}_{I}\right)} \exp \left(-\frac{\gamma_{e}^{\text {th }}}{\bar{g}_{S E} \gamma_{S}}\right)\right]
$$

Proof: Referring to [7], the SOP is defined as

$$
P_{R S}=\operatorname{Pr}\left(\gamma_{D} \geq \gamma_{t}^{\text {th }}, \gamma_{E}<\gamma_{e}^{\text {th }}\right)
$$

It is easy to find that $\gamma_{D}$ and $\gamma_{E}$ are independent with each other. Hence, the SOP can be further expressed as

$$
P_{R S}=\operatorname{Pr}\left(\gamma_{D} \geq \gamma_{t}^{\text {th }}\right) \operatorname{gPr}\left(\gamma_{E}<\gamma_{e}^{\text {th }}\right)=\left(1-P_{c o}\right)\left(1-P_{s o}\right)
$$

Substituting (8) and (12) into (16), the expression in Theorem 3 is readily extracted.

\section{Numerical Results}

In this section, the numerical results are provided to validate the derived expressions. Without loss of generality, we set $\eta=0.8, R_{t}=2 \mathrm{bits} / \mathrm{s} / \mathrm{Hz}, R_{s}=1 \mathrm{bit} / \mathrm{s} / \mathrm{Hz}, \bar{\gamma}_{I}=-15 d B, \bar{\gamma}_{S D}=\bar{\gamma}_{S E}=\bar{\gamma}_{D E}=10 \mathrm{~dB}$. Specifically, we set $\bar{\gamma}_{I}^{\prime}=-45 d B$, which means that an amount of $30 \mathrm{~dB}$ is cancelled through the SIC operation. We note that, this amount of cancellation can be easily achieved by some simple passive SIC operations [13].

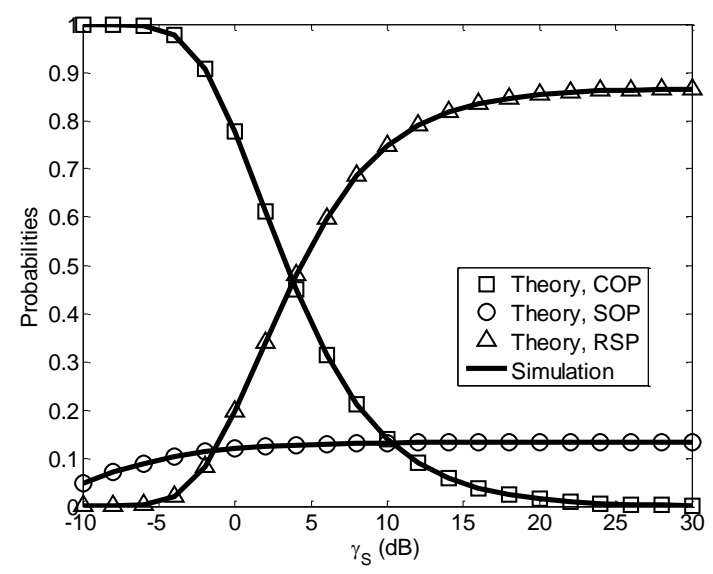

Fig. 3: COP, SOP and RSP vs. the transmit SNR $\gamma_{S}$ with $\beta=0.8$. 
In Fig. 3, the impacts of transmit SNR $\gamma_{S}$ on COP, SOP and RSP are examined. Specifically, we set $\beta=0.8$ and $\bar{\gamma}_{S D}=10 \mathrm{~dB}$. As can be seen, the theoretical results match the numerical simulations very well. From this figure, we see that the COP and RSP are decreasing functions of $\gamma_{S}$ while the SOP is just On the contrary. Also, a phenomenon of performance floor is observed in all three lines.

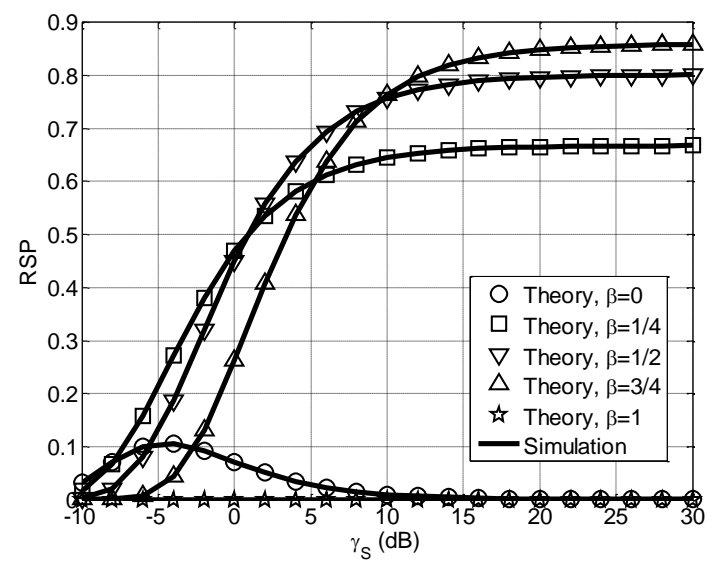

Fig. 4: RSP vs. the transmit SNR $\gamma_{S}$ with different $\beta$.

In Fig. 4, the relationship between the transmit SNR $\gamma_{S}$ and RSP is compared with different $\beta$. We note that, the RSP is rather small when $\beta=0$. This is readily to understand because no energy of the signal is split for EH, so no AN is sent for confusing the eavesdropper. Furthermore, it is observed that the RSP improves rapidly with the increasing of $\beta$. However, it does not means that the larger $\beta$ is, the better the RSP keeps. Actually, it depends on the condition of $\gamma_{S}$. Particularly, the RSP remains zero when $\beta=1$. This is also understandable because in this extreme case, all the energy is used for transmitting AN while no energy is remained for information decoding.

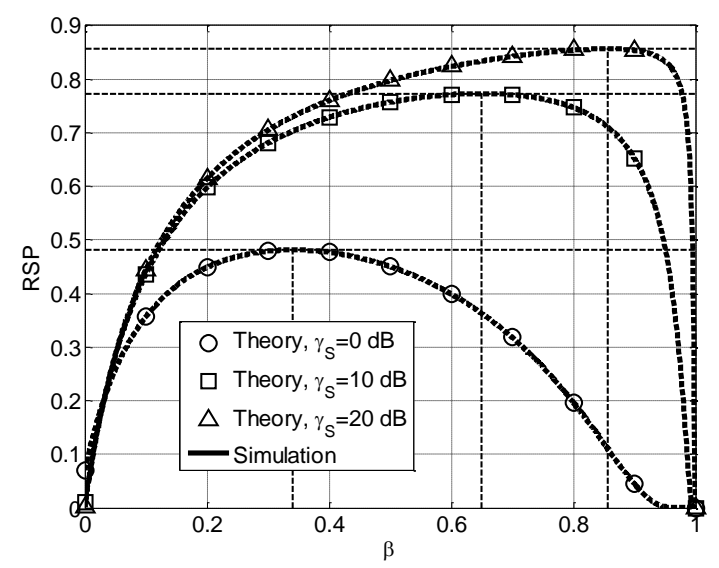

Fig. 5: RSP vs. the power-splitting ratio $\beta$ with different $\gamma_{S}$.

In Fig. 5, the relationship between the power-splitting ratio $\beta$ and RSP is illustrated with different $\gamma_{s}$. In this figure, a significant enhancement is readily found when a larger $\gamma_{s}$ is provided. In addition, for a certain $\gamma_{s}$, the RSP improves with the increasing of $\beta$ at first and then get reversed. Also, the turning point changes with different $\gamma_{s}$. All above observations indicate the existence of an optimum $\beta$.

\section{Conclusion}

This work investigated the secrecy performance of FD SWIPT networks with AN from a PS perspective. The exact closed-form expressions of the COP, SOP and RSP were derived, respectively, and the validation is demonstrated via numerical simulations. The results showed that the power-splitting ratio $\beta$ and the transmit SNR $\gamma_{S}$ have great influence on the secrecy performance. Specifically, the secrecy performance improved rapidly with the increasing of $\beta$. However, whether a larger $\beta$ would result in a better security depends on the condition of $\gamma_{s}$. Also, it was easily found that the secrecy performance is improved with the 
increasing of $\beta$ at first and then gets reversed for a certain $\gamma_{s}$. Furthermore, different values of optimum $\beta$ were derived for various $\gamma_{s}$.

\section{Acknowledgements}

This work is supported by the National Natural Science Foundation of China under Grant Nos. 61371122 and 61471393, the Jiangsu Provincial Natural Science Foundation of China under Grant No. BK20150718, and the Open Research Fund of Key Lab of Broadband Wireless Communication and Sensor Network Technology (Nanjing University of Post and Telecommunications), Ministry of Education under Grant No. NYKL201506.

\section{References}

[1] A. D. Wyner. The wire-tap channel. Bell Sys. Tech. J., 1975, 54(8): 1355-1387.

[2] T. Zhang, Y. Huang, Y. Cai, et al. Secure Multi-Antenna Cognitive Wiretap Networks. IEEE Trans. Veh. Techno., to appear, 2016.

[3] Y. Liu, L. Wang, S. a. R. Zaidi, et al. Secure D2D Communication in Large-Scale Cognitive Cellular Networks: A Wireless Power Transfer Model. IEEE Trans. Commun., 2016, 64(1): 329-342.

[4] Y. Deng, L. Wang, M. Elkashlan, et al. Physical Layer Security in Three-Tier Wireless Sensor Networks: A Stochastic Geometry Approach. IEEE Trans. Inf. Forensics Security, 2016, 11(6): 1128-1138.

[5] C. Wang, H. M. Wang. Opportunistic Jamming for Enhancing Security: Stochastic Geometry Modeling and Analysis. IEEE Trans. Veh. Techno., 2016, 65(12): 10213-10217.

[6] S. Goel, R. Negi. Guaranteeing Secrecy using Artificial Noise. IEEE Trans. Wireless Commun., 2008, 7(6): 21802189.

[7] L. Wang, Y. Cai, Y. Zou, et al. Joint Relay and Jammer Selection Improves the Physical Layer Security in the Face of CSI Feedback Delays. IEEE Trans. Veh. Techno., 2016, 65(8): 6259-6274.

[8] J. Zhang, C. Yuen, C. K. Wen, et al. Large System Secrecy Rate Analysis for SWIPT MIMO Wiretap Channels. IEEE Trans. Inf. Forensics Security, 2016.

[9] Z. Caijun, H. A. Suraweera, Z. Gan, et al. Wireless Information and Power Transfer With Full Duplex Relaying. IEEE Trans. Commun., 2014, 62(10): 3447-3461.

[10] Y. Zeng, R. Zhang. Full-Duplex Wireless-Powered Relay With Self-Energy Recycling. IEEE Wireless Communications Letters, 2015, 4(2): 201-204.

[11] D. Wang, R. Zhang, X. Cheng, et al. Capacity-Enhancing Full-Duplex Relay Networks based on Power Splitting (PS-)SWIPT. IEEE Trans. Veh. Techno., to appear, 2016.

[12] Y. Bi, H. Chen. Accumulate and Jam: Towards Secure Communication via A Wireless-Powered Full-Duplex Jammer. IEEE J. Sel. Topics Signal Process., 2016, 10(8): 1538-1550.

[13] Z. Zhongshan, C. Xiaomeng, L. Keping, et al. Full duplex techniques for $5 \mathrm{G}$ networks: self-interference cancellation, protocol design, and relay selection. IEEE Commun. Mag., May. 2015, 53(5): 128-137. 